KAIST-TH 2001/05

DESY 01-033

\title{
Radion Phenomenology in the Randall Sundrum Scenario
}

\author{
S. Bae, P. Ko, H. S. Lee \\ Department of Physics, KAIST, Taejon, 305-701, Korea \\ E-mail:pko@muon.kaist.ac.kr \\ Jungil Lee \\ Deutsches Elektronen-Synchrotron DESY, D-22603 Hamburg, Germany \\ E-mail: jungil@mail.desy.de
}

\begin{abstract}
Phenomenology of a radion $(\phi)$ in the Randall-Sundrum scenario is discussed. The radion couples to the trace of energy momentum tensor of the standard model with a strength suppressed only by a new scale $\left(\Lambda_{\phi}\right)$ which is an order of the electroweak scale. In particular, the effective coupling of a radion to two gluons is enhanced due to the trace anomaly of QCD. Therefore, its production cross section at hadron colliders could be enhanced, and the dominant decay mode of a relatively light radion is $\phi \rightarrow g g$, unlike the SM Higgs boson case. We also present constraints on the mass $m_{\phi}$ and the new scale $\Lambda_{\phi}$ from the Higgs search limit at LEP, perturbative unitarity bound and the stability of the radion/Higgs potential under radiative corrections.
\end{abstract}




\section{INTRODUCTION}

Despite the tremendous success, the standard model (SM) has several theoretical drawbacks, one of which is related with stabilizing the electroweak scale relative to the Planck scale under quantum corrections, which is known as the gauge hierarchy problem. Traditionally, there have been basically two avenues to solve this problem : (i) electroweak gauge symmetry is spontaneously broken by some new strong interactions (technicolor or its relatives) or (ii) there is a supersymmetry (SUSY) which is spontaneously broken in a hidden sector, and superpartners of SM particles have masses around the electroweak scale $O(100-1000) \mathrm{GeV}$. However, new mechanisms based on the developments in superstring and $\mathrm{M}$ theories including D-branes have been suggested by Randall and Sundrum [1]. If our world is confined to a three-dimensional brane and the warp factor in the Randall and Sundrum (RS) theory is much smaller than 1, then loop corrections cannot destroy the mass hierarchy derived from the relation $v=e^{-k r_{c} \pi} v_{0}$, where $v_{0}$ is the VEV of Higgs field $\left(\sim O\left(M_{P}\right)\right)$ in the 5-dimensional RS theory, $e^{-k r_{c} \pi}$ is the warp factor, and $v$ is the VEV of Higgs field $(\sim 246 \mathrm{GeV})$ in the 4-dimensional effective theory of the RS theory by a kind of dimensional reduction. Especially the extra-dimensional subspace need not be a circle $S^{1}$ like the Kaluza-Klein theory [1], and in that case, it is crucial to have a mechanism to stabilize the modulus. One such a mechanism was recently proposed by Goldberger and Wise [2,3], and also by Csáki et al. [4]. In such a case, the modulus (or the radion $\phi$ from now on) is likely to be lighter than the lowest Kaluza-Klein excitations of bulk fields. Also its couplings to the SM fields are completely determined by general covariance in the fourdimensional spacetime, as shown in Eq. (1) below. If this scenario is realized in nature, this radion could be the first signature of this scenario, and it would be important to determine its phenomenology at the current/future colliders, which is the purpose of this talk [5]. Some related issues were addressed in Refs. [6,7].

In this talk, we first recapitulate the interaction Lagrangian for a single radion and the SM fields in the Randall Sundrum scenario, and discuss the decay rates and the branching 
ratios of the radion into SM particles. Then the perturbative unitarity bounds and stability condition for the radion Higgs potential on the radion mass $m_{\phi}$ and $\Lambda_{\phi}$ are considered. Current bounds on the SM Higgs search can be easily translated into the corresponding bounds on the radion. Then the radion production cross sections at next linear colliders (NLC's) and hadron colliders such as the Tevatron and LHC are considered. Then our results will be summarized at the end.

\section{RADION IN THE RANDALL SUNDRUM SCENARIO I}

In the RS theory I [1], the hierarchy problem between the Planck scale $\Lambda_{\mathrm{Pl}} \sim 10^{18} \mathrm{GeV}$ and the electroweak scale $\Lambda_{\mathrm{EW}} \sim 10^{2} \mathrm{GeV}$ can be solved geometrically via $v=e^{-k r_{c} \pi} v_{0}$ with $k r_{c} \sim 12$, where the warp factor $e^{-k r_{c} \pi}$ is in the classical RS metric,

$$
d s_{\mathrm{RS}}^{2}=e^{-2 k r_{c}|y|} \eta_{\mu \nu} d x^{\mu} d x^{\nu}-r_{c}^{2} d y^{2}
$$

The radius $r_{c}$ is the $\mathrm{VEV}$ of the $y y$-component of metric $G_{a b}(x, y)$. When the bulk field $G_{y y}(x, y)$ is decomposed into Kaluza-Klein (KK) modes, the lowest lying mode is the massless radion $(\phi)$ in the original RS model I, since there is no tree-level stabilization mechanism for the radion. The loop corrections can solve the hierarchy problem, but give too light radion to be consistent with experiments [8]. Therefore, a tree-level stabilization mechanism is needed, and the Goldberger-Wise mechanism [2] is a promising one, because they stabilized the modulus without any severe fine-tuning of the parameters in the full theory.

In the Goldberger-Wise stabilization mechanism, there is a bulk scalar field $\Phi(x, y)$ which has large quartic self-interactions only on the hidden and visible branes, and an extradimension dependent $\operatorname{VEV} \tilde{\Phi}(y)$. After replacing the field $\Phi(x, y)$ in the original Lagrangian with its $\operatorname{VEV} \tilde{\Phi}(y)$ and integrating the Lagrangian over $y$, we have the modulus stabilizing potential. Since there is a potential stabilizing the radion, the radion has a mass of order $O(10) \mathrm{GeV}$ at the tree level [3]. From a recent analysis [9], it was known that the one-loop allowed radion mass can be $\sim O(10)$ times larger than the tree-level one, but the radion is still lighter than the Kaluza-Klein modes. 


\section{SCALE ANOMALY AND THE INTERACTION LAGRANGIAN FOR A RADION}

\section{A. Scale Anomaly}

If a Lagrangian possesses no dimensionful parameter, there is a scale symmetry at classical level, for which one can construct a conserved Noether current $D_{\mu}$. One can show that the improved energy momentum tensor $\Theta_{\mu \nu}$ satisfies the following relation :

$$
\partial_{\mu} D^{\mu}=\Theta_{\mu}^{\mu}
$$

For example, if one considers QCD as an example, the improved energy momentum tensor $\Theta^{\mu \nu}$ is given by

$$
\begin{aligned}
\Theta^{\mu \nu} & =-F^{a \lambda \mu} F_{\lambda}^{a \nu}+\frac{1}{4} g^{\mu \nu} F^{a \rho \sigma} F_{\rho \sigma}^{a} \\
& +\frac{i}{2} \bar{q}\left(\gamma^{\mu} D^{\nu}+\gamma^{\nu} D^{\mu}\right) q-g^{\mu \nu} \bar{q}\left(i \gamma^{\alpha} D_{\alpha}-m_{q}\right) q .
\end{aligned}
$$

It is clear that this theory has traceless energy momentum tensor at classical level if we consider the massless quark limit (without dimensionful parameters).

However, this is no longer true in quantum field theory (QFT) in which the radiative corrections are usually divergent so that renormalization procedure is called for. In the renormalization, one needs to regularize the theory with a suitable cutoff parameter to make loop integrals finite. Therefore a hidden cutoff scale is necessarily involved with QFT. If we use the dimensional regularization instead of cutoff regularization, the dimensionless parameter in four-dimensional theory is no longer dimensionless in $D$-dimensional theory. And the classical scale symmetry in $4-D$ is no longer a good symmetry in arbitrary $D$ dimensions.

To look into the energy momentum tensor $\Theta_{\mu \nu}$ more closely at quantum level, let us

consider the scale dependence of quantum effective action. Since the renormalized coupling $g_{s}(\mu)$ has a scale dependence, 


$$
\mu \frac{\partial g_{s}}{\partial \mu}=\beta\left(g_{s}\right)
$$

one finds that

$$
\mu \frac{\partial \mathcal{L}}{\partial \mu}=\beta\left(g_{s}\right) \frac{\partial \mathcal{L}}{\partial g_{s}}=\Theta_{\mu}^{\mu}
$$

Therefore we end up with

$$
\Theta_{\mu}^{\mu}(\mathrm{SM})^{\mathrm{anom}}=\sum_{G=\mathrm{SU}(3)_{C}, \cdots} \frac{\beta_{G}\left(g_{G}\right)}{2 g_{G}} \operatorname{tr}\left(F_{\mu \nu}^{G} F^{G \mu \nu}\right) .
$$

Since the classical scale symmetry is broken by quantum effects, it is called scale anomaly

(like the chiral anomaly) [10]. If there were mass parameters in the classical Lagrangian, the scale symmetry would have been broken already at classical level so that $\Theta_{\mu}^{\mu}$ is not zero and this should be considered altogether with the scale anomaly term.

\section{B. Interaction Lagrangian for a Radion}

The interaction of the radion with the SM fields at an electroweak scale is dictated by the 4-dimensional general covariance, and is described by the following effective Lagrangian via canonical normalizations of the fields [4, 3 ] :

$$
\mathcal{L}_{\text {int }}=\frac{\phi}{\Lambda_{\phi}} \Theta_{\mu}^{\mu}(\mathrm{SM})+\ldots
$$

where $\Lambda_{\phi}=\langle\phi\rangle \sim O(v)$. The radion becomes massive after the modulus stabilization, and its mass $m_{\phi}$ is a free parameter of electroweak scale [4]. Therefore, two parameters $\Lambda_{\phi}$ and $m_{\phi}$ are required in order to discuss productions and decays of the radion at various settings. The couplings of the radion with the SM fields look like those of the SM Higgs, except for $v \rightarrow \Lambda_{\phi}$. However, there is one important thing to be noticed : the quantum corrections to the trace of the energy-momentum tensor lead to trace anomaly, leading to the additional effective radion couplings to gluons or photons in addition to the usual loop contributions. This trace anomaly contributions will lead to distinct signatures of the radion compared to the SM Higgs boson. 
The trace of energy-momentum tensor of the SM fields at tree level is easily derived :

$$
\begin{aligned}
\Theta_{\mu}^{\mu}(\mathrm{SM})^{\text {tree }} & =\sum_{f} m_{f} \bar{f} f-2 m_{W}^{2} W_{\mu}^{+} W^{-\mu}-m_{Z}^{2} Z_{\mu} Z^{\mu} \\
& +\left(2 m_{h}^{2} h^{2}-\partial_{\mu} h \partial^{\mu} h\right)+\ldots,
\end{aligned}
$$

where we showed terms with only two SM fields, since we will discuss two body decay rates of the radion into the SM particles, except the gauge bosons of which virtual states are also considered. The couplings between the radion $\phi$ and a fermion pair or a weak gauge boson pair are simply related with the SM Higgs couplings with these particles through simple rescaling : $g_{\phi-f-\bar{f}}=g_{h-f-\bar{f}}^{\mathrm{SM}} v / \Lambda_{\phi}$, and so on. On the other hand, the $\phi-h-h$ coupling is more complicated than the SM $h-h-h$ coupling. There is a momentum dependent part from the derivatives acting on the Higgs field, and this term can grow up as the radion mass gets larger or the CM energy gets larger in hadroproductions of the radion. It may lead to the violation of perturbative unitarity, which will be addressed after we discuss the decay rates of the radion. Finally, there is no $h-\phi-\phi$ coupling, since the radion couples to the trace of the energy momentum tensor and there should be no $h-\phi$ mixing after field redefinitions in terms of physical fields.

In addition to the tree-level $T_{\mu}^{\mu}(\mathrm{SM})^{\text {tree }}$, there is also the trace anomaly term for gauge fields [10], Eq. (60). This trace anomaly couples with the parameter of conformal transformations in our 3-brane. And the radion $\phi$ plays the same role as the parameter of conformal transformation, since it belongs to the warp factor in the 5 dimensional RS metric [4. Therefore, the parameter associated with the conformal transformation is identified with the radion field $\phi$. As a result, the radion $\phi$ has a coupling to the trace anomaly term. For QCD sector as an example, one has

$$
\frac{\beta_{Q C D}}{2 g_{s}}=-\left(11-\frac{2}{3} n_{f}\right) \frac{\alpha_{s}}{8 \pi} \equiv-\frac{\alpha_{s}}{8 \pi} b_{Q C D},
$$

where $n_{f}=6$ is the number of active quark flavors. There are also counterparts in the $S U(2) \times U(1)$ sector. This trace anomaly has an important phenomenological consequence. 
For a relatively light radion, the dominant decay mode will not be $\phi \rightarrow b \bar{b}$ as in the SM Higgs, but $\phi \rightarrow g g$.

\section{RADION PHENOMENOLOGY}

\section{A. Decay Properties of a Radion}

Using the interaction Lagrangian Eq. (7), it is straightforward to calculate the decay rates and branching ratios of the radion $\phi$ into $f \bar{f}, W^{+} W^{-}, Z^{0} Z^{0}, g g$ and $h h$.

$$
\begin{aligned}
\Gamma(\phi \rightarrow f \bar{f}) & =N_{c} \frac{m_{f}^{2} m_{\phi}}{8 \pi \Lambda_{\phi}^{2}}\left(1-x_{f}\right)^{3 / 2}, \\
\Gamma\left(\phi \rightarrow W^{+} W^{-}\right) & =\frac{m_{\phi}^{3}}{16 \pi \Lambda_{\phi}^{2}} \sqrt{1-x_{W}}\left(1-x_{W}+\frac{3}{4} x_{W}^{2}\right), \\
\Gamma(\phi \rightarrow Z Z) & =\frac{m_{\phi}^{3}}{32 \pi \Lambda_{\phi}^{2}} \sqrt{1-x_{Z}}\left(1-x_{Z}+\frac{3}{4} x_{Z}^{2}\right), \\
\Gamma(\phi \rightarrow h h) & =\frac{m_{\phi}^{3}}{32 \pi \Lambda_{\phi}^{2}} \sqrt{1-x_{h}}\left(1+\frac{x_{h}}{2}\right)^{2}, \\
\Gamma(\phi \rightarrow g g) & =\frac{\alpha_{s}^{2} m_{\phi}^{3}}{32 \pi^{3} \Lambda_{\phi}^{2}}\left|b_{Q C D}+\sum_{q} I_{q}\left(x_{q}\right)\right|^{2},
\end{aligned}
$$

where $x_{f, W, Z, h}=4 m_{f, W, Z, h}^{2} / m_{\phi}^{2}$, and $I(z)=z[1+(1-z) f(z)]$ with

$$
\begin{aligned}
f(z) & =-\frac{1}{2} \int_{0}^{1} \frac{d y}{y} \ln \left[1-\frac{4}{z} y(1-y)\right] \\
& =\left\{\begin{array}{cl}
\arcsin ^{2}(1 / \sqrt{z}), & z \geq 1, \\
-\frac{1}{4}\left[\ln \left(\frac{1+\sqrt{1-z}}{1-\sqrt{1-z}}\right)-i \pi\right]^{2}, & z \leq 1 .
\end{array}\right.
\end{aligned}
$$

Note that as $m_{t} \rightarrow \infty$, the loop function approaches $I\left(x_{t}\right) \rightarrow 2 / 3$ so that the top-quark effect decouples and one is left with $b_{Q C D}$ with $n_{f}=5$. For $\phi \rightarrow W W, Z Z$, we have ignored $S U(2)_{L} \times U(1)_{Y}$ anomaly, since these couplings are allowed at the tree-level already, unlike the $\phi-g-g$ or $\phi-\gamma-\gamma$ couplings. This should be a good approximation for a relatively light radion.

Using the above results, we show the decay rate of the radion and the relevant branching ratio for each channel available for a given $m_{\phi}$ in Figs. 11 and 2 . 


\section{FIGURES}

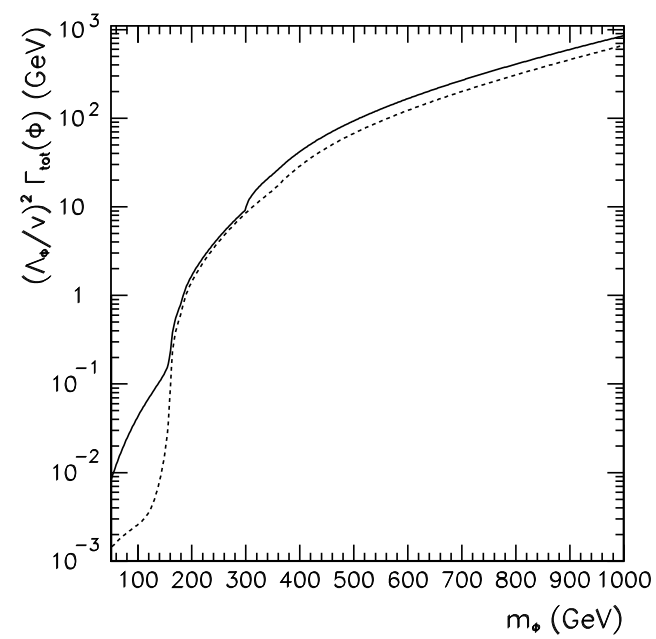

FIG. 1. The total decay rate (in $\mathrm{GeV}$ ) for the radion $\phi$ for $m_{h}=150 \mathrm{GeV}$ with a scale factor $\left(\Lambda_{\phi} / v\right)^{2}$. The decay rate of the SM Higgs boson is shown in the dashed curve for comparison.

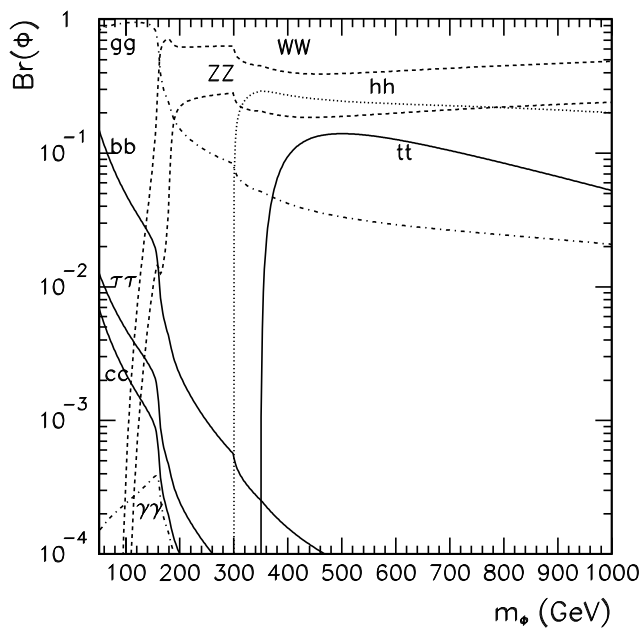

FIG. 2. The branching ratios for the radion $\phi$ into the SM particles.

In the numerical analysis, we use $\Lambda_{\phi}=v=246 \mathrm{GeV}$ and $m_{h}=150 \mathrm{GeV}$, and also included the QCD corrections. The decay rates for different values of $\Lambda_{\phi}$ can be obtained through the following scaling : $\Gamma\left(\Lambda_{\phi}\right)=\left(v / \Lambda_{\phi}\right)^{2} \Gamma\left(\Lambda_{\phi}=v\right)$. The decay rate scales as $\left(v / \Lambda_{\phi}\right)^{2}$, but the branching ratios are independent of $\Lambda_{\phi}$. In Fig. 11, we also show the decay rate of the SM Higgs boson with the same mass as $\phi$. We note that the light radion with $\Lambda_{\phi}=v$ 
could be a much broader resonance compared to the SM Higgs even if $m_{\phi} \lesssim 2 m_{W}$. This is because the dominant decay mode is $\phi \rightarrow g g$ (see Fig. 2), unlike the SM Higgs for which the $b \bar{b}$ final state is a dominant decay mode. This phenomenon is purely a quantum field theoretical effect : enhanced $\phi-g-g$ coupling due to the trace anomaly. For a heavier radion, it turns out that $\phi \rightarrow V V$ with $V=W$ or $Z$ dominates other decay modes once it is kinematically allowed. Also the branching ratio for $\phi \rightarrow h h$ can be also appreciable if it is kinematically allowed. This is one of the places where the difference between the SM and the radion comes in. If $\Lambda_{\phi} \gg v$, the radion would be a narrow resonance and should be easily observed as a peak in the two jets or $W W(Z Z)$ final states. Especially $\phi \rightarrow Z Z \rightarrow(l \bar{l})\left(l^{\prime} \bar{l}^{\prime}\right)$ will be a gold-plated mode for detecting the radion as in the case of the SM Higgs. Even in this channel, one can easily distinguish the radion from the SM Higgs by difference in their decay width.

\section{B. Perturbative Unitarity}

The perturbative unitarity can be violated (as in the SM) in the $V_{L} V_{L} \rightarrow V_{L} V_{L}$ or $h h \rightarrow V_{L} V_{L}$, etc. Here we consider $h h \rightarrow h h$, since the $\phi-h-h$ coupling scales like $s / \Lambda_{\phi}$ for large $s \equiv\left(p_{h_{1}}+p_{h_{2}}\right)^{2}$. The tree-level amplitude for this process is

$$
\begin{aligned}
\mathcal{M}(h h \rightarrow h h)= & -6 \lambda-\frac{1}{\Lambda_{\phi}^{2}}\left(\frac{s^{2}}{s-m_{\phi}^{2}}+\frac{t^{2}}{t-m_{\phi}^{2}}+\frac{u^{2}}{u-m_{\phi}^{2}}\right) \\
& -36 \lambda^{2} v^{2}\left(\frac{1}{s-m_{h}^{2}}+\frac{1}{t-m_{h}^{2}}+\frac{1}{u-m_{h}^{2}}\right)
\end{aligned}
$$

where $\lambda$ is the Higgs quartic coupling, and $s+t+u=4 m_{h}^{2}$. Projecting out the $J=0$ partial wave component $\left(a_{0}\right)$ and imposing the partial wave unitarity condition $\left|a_{0}\right|^{2} \leq \operatorname{Im}\left(a_{0}\right)$ (i.e. $\left.\left|\operatorname{Re}\left(a_{0}\right)\right| \leq 1 / 2\right)$, we get the following relation among $m_{h}, v, m_{\phi}$ and $\Lambda_{\phi}$, for $s \gg m_{h}^{2}, m_{\phi}^{2}$ :

$$
\left|\frac{2 m_{h}^{2}+m_{\phi}^{2}}{8 \pi \Lambda_{\phi}^{2}}+\frac{3 \lambda}{8 \pi}\right| \leq \frac{1}{2} .
$$

This bound is shown in the lower three curves of Fig. 3. We note that the perturbative unitarity is broken for relatively small $\Lambda_{\phi} \lesssim 130(300) \mathrm{GeV}$ for $m_{\phi} \sim 200 \mathrm{GeV}(1 \mathrm{TeV})$. 
Therefore, the tree level results should be taken with care for this range of $\Lambda_{\phi}$ for a given radion mass.

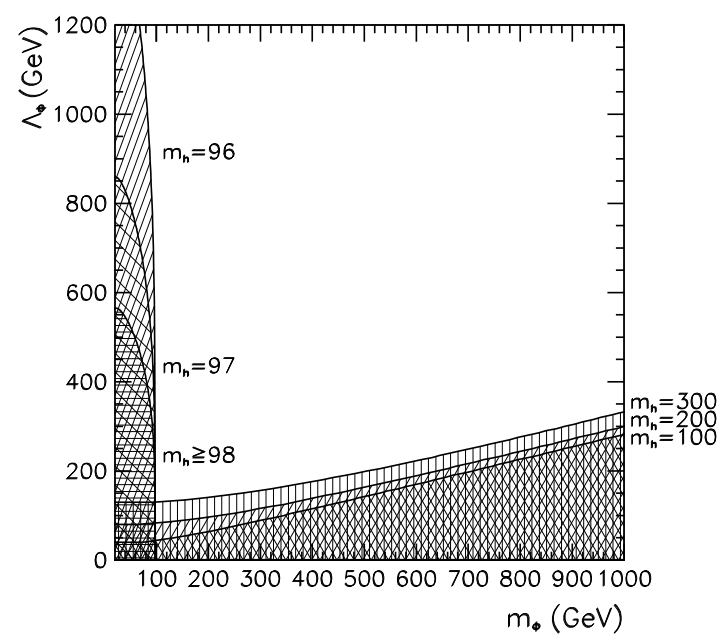

FIG. 3. The excluded region in the $m_{\phi}$ and $\Lambda_{\phi}$ space obtained from the recent L3 result on the SM Higgs search (the left three curves) and perturbative unitarity bound (the lower three curves).

\section{Stability of the radion and Higgs potential under radiative corrections}

Adding the loop corrections from the scalar, fermion vector and KK-mode sectors, the final effective potential up to the one-loop level [9] is

$$
\begin{aligned}
& V_{\text {eff }}(h, \phi) \\
= & V_{\text {tree }}(h, \phi)+V_{1 \text { loop }}^{\mathrm{KK}}(\phi)+V_{1 \text { loop }}^{\text {scalar }}(h, \phi)+V_{1 \text { loop }}^{\text {fermion }}(h)+V_{1 \text { loop }}^{\text {vector }}(h) \\
= & V_{\phi}(\phi)+\frac{\lambda}{4}\left(h^{2}-v_{0}^{2} e^{-2 \phi / \Lambda_{\phi}}\right)^{2}+\delta V_{\mathrm{TeV}} e^{-4 \phi / \Lambda_{\phi}} \\
+ & \frac{1}{4(4 \pi)^{2}}\left\{\lambda^{2}\left(3 h^{2}-v_{0}^{2} e^{-2 \phi / \Lambda_{\phi}}\right)^{2}\left(\log \left[\lambda\left(3 h^{2}-v_{0}^{2} e^{-2 \phi / \Lambda_{\phi}}\right) / \mu^{2}\right]-\frac{3}{2}\right)\right\} \\
& \left.\quad+3 \lambda^{2}\left(h^{2}-v_{0}^{2} e^{-2 \phi / \Lambda_{\phi}}\right)^{2}\left(\log \left[\lambda\left(h^{2}-v_{0}^{2} e^{-2 \phi / \Lambda_{\phi}}\right) / \mu^{2}\right]-\frac{3}{2}\right)\right\} \\
+ & \frac{1}{(4 \pi)^{2}}\left\{-3 T^{2}\left(\log \frac{T}{\mu^{2}}-\frac{3}{2}\right)+\frac{3}{2} W^{2}\left(\log \frac{W}{\mu^{2}}-\frac{5}{6}\right)+\frac{3}{4} Z^{2}\left(\log \frac{Z}{\mu^{2}}-\frac{5}{6}\right)\right\} .
\end{aligned}
$$

The procedure to determine $m_{\phi}$ and $\Lambda_{\phi}$ is to find a parameter point of $\left(v_{0}, v_{v}, v_{h}, m, \delta V_{\mathrm{TeV}}\right)$ which gives a stable vacuum satisfying the two constraints, warp factor $e^{-k r_{c} \pi}=O\left(M_{W} / M_{P}\right)$ 
and Higgs $\mathrm{VEV} v \simeq 246 \mathrm{GeV}$ and next determine the values of $m_{\phi}$ and $\Lambda_{\phi}$ from the relations to the previous parameters.

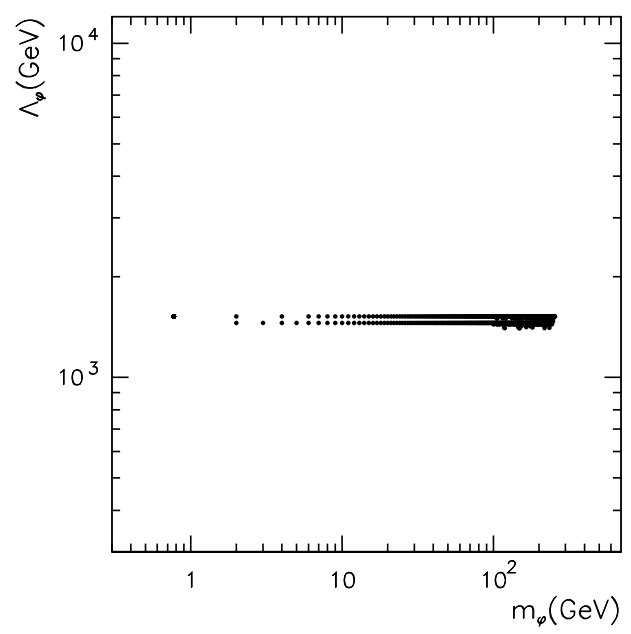

FIG. 4. The allowed points for the one-loop effective potential $\left(M_{(5)}=0.8 M_{\mathrm{Pl}}\right.$ and $m_{h}=125$ $\mathrm{GeV})$.

For the one-loop effective potential, the allowed region for the 5-dimensional Planck mass $M_{(5)}=0.8 M_{\mathrm{Pl}}$ and the Higgs mass $m_{h}=125 \mathrm{GeV}$ is $0.8 \mathrm{GeV} \lesssim m_{\phi} \lesssim 260 \mathrm{GeV}$ and $1400 \mathrm{GeV} \lesssim \Lambda_{\phi} \lesssim 1500 \mathrm{GeV}$ from Fig. 田 (the central value of $k b_{0} / 2$ is still about 36 ). Almost all the data are focused at $\Lambda_{\phi}=1490 \mathrm{GeV}$ like the tree level case. Therefore, we can conclude that the naturally allowed regions are similar for the tree and one-loop cases, and there is a small shift of the central value of $\Lambda_{\phi}$ (or equivalently $k b_{0} / 2$ ) due to the oneloop corrections. When $\delta V_{\mathrm{TeV}}^{\mathrm{KK}}$ and other parameters are changed continuously, the allowed region in Fig. 4 can be broader in $\Lambda_{\phi}$. From numerical analysis, we have found that only the negative values of $\delta V_{\mathrm{TeV}}^{\mathrm{KK}}$ can be allowed. Negative values of the tension shift $\delta V_{\mathrm{TeV}}^{\mathrm{KK}}$ produce many parameter points which were not allowed at the tree level, and considerable parts of these new points make the radion mass sufficiently larger than the tree-level upper bound of the mass. It is phenomenologically noteworthy that the one-loop upper bound of the radion mass $m_{\phi}$ is rather larger than the tree-level one by about five times. But the radion is still the first signal of the RS theory lighter than the lowest-lying KK mode with a mass of order 
$O(1) k e^{-k b_{0} / 2} \simeq 0.8 \Lambda_{\phi}\left[11\right.$, ,12], because the radion mass is smaller than about $\Lambda_{\phi} / 6$. Due to $m_{\phi} \lesssim 260 \mathrm{GeV}$, the branching ratios of the radion into gluon or $W$ boson pairs are dominant according to the discussions in the previous subsection.

\section{Radion Productions at hadron and linear colliders}

The production cross sections of the radion at hadron colliders are given by the gluon fusion into the radion through quark-loop diagrams, as in the case of Higgs boson production, and also through the trace anomaly term, Eq. (4), which is not present in the case of the SM Higgs boson :

$$
\sigma(p p(\operatorname{or} p \bar{p}) \rightarrow \phi X)=K \hat{\sigma}_{\mathrm{LO}}(g g \rightarrow \phi) \int_{\tau}^{1} \frac{\tau}{x} g\left(x, Q^{2}\right) g\left(\tau / x, Q^{2}\right) d x
$$

where $\tau \equiv m_{\phi}^{2} / s$ and $\sqrt{s}$ is the CM energy of the hadron colliders $(\sqrt{s}=2 \mathrm{TeV}$ and $14 \mathrm{TeV}$ for the Tevatron and LHC, respectively). The $K$ factor includes the QCD corrections, and we set $K=1.5$. The parton-level cross section for $g g \rightarrow \phi$ is given by

$$
\hat{\sigma}_{\mathrm{LO}}(g g \rightarrow \phi)=\frac{\alpha_{s}^{2}(Q)}{256 \pi \Lambda_{\phi}^{2}}\left|b_{Q C D}+\sum_{q} I_{q}\left(x_{q}\right)\right|^{2}
$$

where $I(z)$ is given in the Eq. (11). For the gluon distribution function, we use the CTEQ5L parton distribution functions [13].

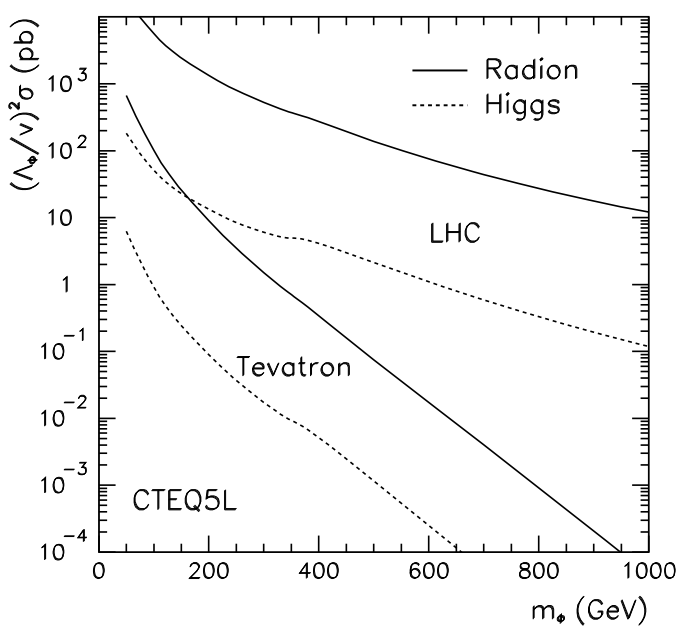


FIG. 5. The radion production cross section via gluon fusions at the Tevatron $(\sqrt{s}=2 \mathrm{TeV})$ and LHC $(\sqrt{s}=14 \mathrm{TeV})$ with a scale factor $\left(\Lambda_{\phi} / v\right)^{2}$. The Higgs production cross sections are shown in dashed curves for comparison.

In Fig. 5, we show the radion production cross sections at the Tevatron and LHC as functions of $m_{\phi}$ for $\Lambda_{\phi}=v$. We set the renormalization scale $Q=m_{\phi}$ as shown in the figure. When we vary the scale $Q$ between $m_{\phi} / 2$ and $2 m_{\phi}$, the production cross section changes about $+30 \%$ to $-20 \%$. The production cross section will scale as $\left(v / \Lambda_{\phi}\right)^{2}$ as before. Compared to the SM Higgs boson productions, one can clearly observe that the trace anomaly can enhance the hadroproductions of a radion enormously. As in the SM Higgs boson, there is a great possibility to observe the radion up to mass $\sim 1 \mathrm{TeV}$ if $\Lambda_{\phi} \sim v$. For a smaller $\Lambda_{\phi}$, the cross section becomes larger but the radion becomes very broader and it becomes more difficult to find such a scalar. For a larger $\Lambda_{\phi}$, the situation becomes reversed : the smaller production cross section, but a narrower width resonance, which is easier to detect. In any case, however, one has to keep in mind that the perturbative unitarity may be violated in the low $\Lambda_{\phi}$ region.

At the $e^{+} e^{-}$colliders, the main production mechanism for the radion $\phi$ is the same as the SM Higgs boson : the radion-strahlung from $Z^{0}$ and the $W W$ fusion, the latter of which becomes dominant for a larger CM energy [14. Again we neglect the anomaly contributions here. Since both of these processes are given by the rescaling of the SM Higgs production rates, we can use the current search limits on Higgs boson to get the bounds on the radion. With the data from L3 collaboration [15], we show the constraints of $\Lambda_{\phi}$ and $m_{\phi}$ in the left three curves of Fig. 3. Since L3 data is for $\sqrt{s}=189 \mathrm{GeV}$ and the mass of $Z$ boson is about $91 \mathrm{GeV}$, the allowed energy for a scalar particle is about $98 \mathrm{GeV}$. If the mass of the scalar particle is larger than $98 \mathrm{GeV}$, then the cross section vanishes. Therefore, if $m_{\phi}$ is larger than $98 \mathrm{GeV}$, there is no constraint on $\Lambda_{\phi}$. And the forbidden region in $m_{\phi}-\Lambda_{\phi}$ plane is not changed by $m_{h} \gtrsim 98 \mathrm{GeV}$, because there is no Higgs contribution to the constraint for $m_{h} \gtrsim 98 \mathrm{GeV}$. 


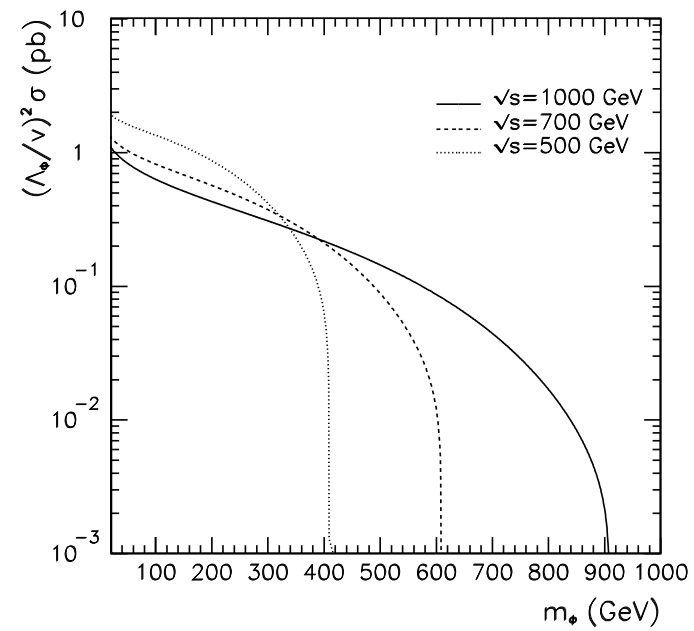

FIG. 6. The production cross section for the radion at NLC's at $\sqrt{s}=500,700$ and 1000 $\mathrm{GeV}$, respectively.

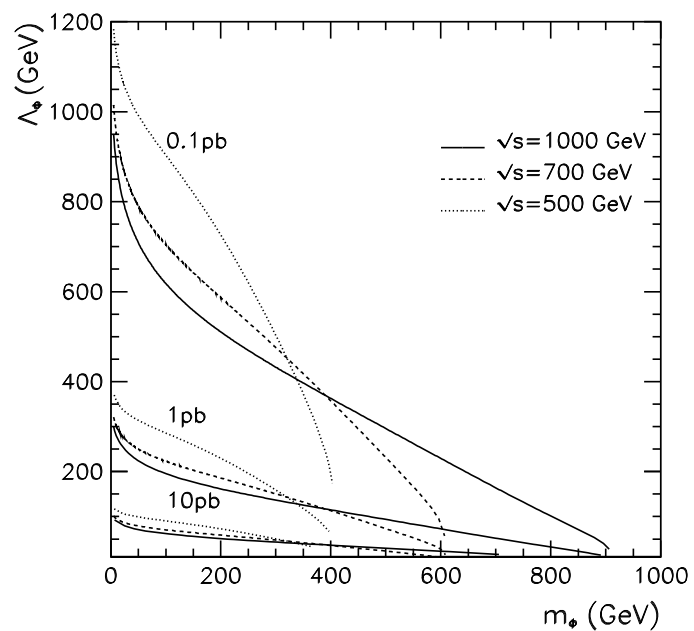

FIG. 7. The constant production cross section curves at next linear colliders (NLC's) for $\sqrt{s}$ $=500,700$ and $1000 \mathrm{GeV}$

The radion production cross sections at NLC's and the corresponding constant production cross section curves in the $\left(\Lambda_{\phi}, m_{\phi}\right)$ plane are shown in Fig. 6 and Fig. 7, respectively. We have chosen three different CM energies for NLC's : $\sqrt{s}=500 \mathrm{GeV}, 700 \mathrm{GeV}$ and $1 \mathrm{TeV}$. We observe that the relatively light radion $\left(m_{\phi} \lesssim 500 \mathrm{GeV}\right)$ with $\Lambda_{\phi} \sim v($ up to $\sim 1 \mathrm{TeV})$ could be probed at NLC's if one can achieve high enough luminosity, since the production cross section in this region is less than a picobarn. 
There are also studies of the radion effects on low energy phenomenology such as muon $(g-2)$ [6] and the weak mixing angle [16]. The effects are generally small in the region where perturbative unitarity is not violated.

\section{CONCLUSIONS}

In summary, we demonstrated that the radion that stabilizes the fifth dimensional modulus in the RS I scenario has characteristic signatures at colliders due to the scale anomaly. Were it not for the scale anomaly in QFT, the radion would have behaved exactly the same as the SM Higgs except that the dimensionful parameter $v$ relevant for the SM Higgs is replaced by the radion decay constant $\Lambda_{\phi}$. The radion would have decayed preferentially

into $b \bar{b}$ final state for $m_{\phi}<2 m_{W}$, and into $W W / Z Z$ for a heavier radion $\left(m_{\phi}>2 m_{V=W, Z}\right)$, like the SM Higgs boson. Because of the scale anomaly, however, the situation drastically changes and $\phi \rightarrow g g$ is greatly enhanced over $\phi \rightarrow b \bar{b}$, and could be a dominant decay channel for a light radion. Also this enhanced $\phi-g-g(\phi-\gamma-\gamma)$ coupling makes the gluon (photon) fusion into a radion the dominant radion production mechanism at hadron (photon) colliders.

\section{ACKNOWLEDGMENTS}

This work was supported in part by BK21 program (HSL), DFG-KOSEF exchange program (PK) and by KOSEF SRC program through CHEP at Kyungpook National University $(\mathrm{PK})$. 


\section{REFERENCES}

[1] L. Randall and R. Sundrum, Phys. Rev. Lett 83, 3370 (1999).

[2] W.D. Goldberger and M.B. Wise, Phys. Rev. Lett. 83, 4922 (1999).

[3] W.D. Goldberger and M.B. Wise, Phys. Lett. B475, 275 (2000).

[4] C. Csáki, M. Graesser, L. Randall and J. Terning, Phys. Rev. D62, 045015 (2000).

[5] S. Bae, P. Ko, Hong Seok Lee, Jungil Lee, Phys. Lett. B487, 299 (2000).

[6] U. Mahanta and S. Rakshit, Phys. Lett. B480, 176 (2000).

[7] G. F. Giudice, R. Rattazzi and J. D. Wells, Nucl. Phys. B595, 250 (2001) ; U. Mahanta and A. Datta, Phys. Lett. B483, 196 (2000) ; K. Cheung, Phys. Rev. D63 056007 (2001).

[8] J. Garriga, O. Pujolàs and T. Tanaka, hep-th/0004109 ; W.D. Goldberger and I.Z. Rothstein, Phys. Lett. B491, 339 (2000).

[9] S. Bae and H. S. Lee, KAIST-TH-2000-13, hep-ph/0011275, to appear in Phys. Lett. B.

[10] R. Crewther, Phys. Rev. Lett. 28, 1421 (1972) ; M. Chanowitz and J. Ellis, Phys. Lett. B40, 397 (1972) ; Phys. Rev. D7, 2490 (1973) ; J. Collins, L. Duncan and S. Joglekar, Phys. Rev. D16, 438 (1977).

[11] W. D. Goldberger and M. B. Wise, Phys. Rev. D60, 107505 (1999).

[12] T. Gherghetta and A. Pomarol, Nucl. Phys. B586 141 (2000).

[13] H.L. Lai et al. (CTEQ Collboration), Eur.Phys. J. C12 375 (2000).

[14] E. Accomando et al., Phys. Rep. 299, 1 (1998).

[15] L3 Collaboration, hep-ex/9909004.

[16] Jihn E. Kim, B. Kyae and J. D. Park, SNUTP-00-017, hep-ph/0007008. 